\title{
Dual Arginine and Glutamic Amino Acids Delivery Effectiveness of Injectable Chitosan-Poloxamer P407 towards Wound Healing Application
}

\author{
Dinh Trung Nguyen ${ }^{1,2}$, Le Hang Dang ${ }^{1,2}$, Bich Tram Nguyen ${ }^{3}$, and Ngoc Quyen Tran ${ }^{1,2 *}$ \\ ${ }^{1}$ Graduate University of Science and Technology, VAST, Ho Chi Minh City, Vietnam \\ ${ }^{2}$ Institute of Applied Materials Science, VAST, Ho Chi Minh City, Vietnam \\ ${ }^{3}$ Department of Natural Science, Thu Dau Mot University, Binh Duong Province, Vietnam
}

*Corresponding author: Ngoc Quyen Tran, Graduate University of Science and Technology, VAST, Ho Chi Minh City, Vietnam; Institute of Applied Materials Science, VAST, Ho Chi Minh City, Vietnam; E-mail: tnquyen@iams.vast.vn

Received: August 12, 2021; Accepted: September 03, 2021; Published: September 05, 2021

\begin{abstract}
The development of bioactive hydrogels has received much attention in the field of tissue regeneration. In the study, we utilized an injectable and biocompatible chitosan-Poloxamer P407 (CS-P407) hydrogel to deliver dual amino acids: glutamic and arginine. The amphiphilic CS-P407 copolymer structure was identified by ${ }^{1} \mathrm{H}-\mathrm{NMR}$ and FITR. The obtained copolymer solution shows the sol-gel transition point at body temperature $\left(35-37^{\circ} \mathrm{C}\right)$, which is suitable for wound healing application. Through SEM imaging, this hydrogel presented a well-defined three-dimensional microporous network. In addition, CS-P407 exposed excellent bio-compatibility, with 90\% fibroblast cell survival. The encapsulation of Arg and/or Glu did not induce any change to sol-gel transition behavior of CS-P407 as well as their biodegradation. The release of Arg and Glu from CS-P407 performed a sustainable profile following the non-Fickian kinetic model. The bioactive hydrogel may provide great potential for future clinical chronic wound management.
\end{abstract}

Keywords: Arginine, Chitosan, Glutamic, Hydrogel, Poloxamer P407, Wound healing

\section{Introduction}

Currently, the engineered bio-materials have significantly contributed to the field of tissue regeneration [1]. Specifically, engineered biomaterials, can control, regulate or mimic the natural regeneration process of tissue or organ. During this process, engineered biomaterials provide the framework structure to promote the migration and the proliferation of the target cells resulting in the promotion of the re-programming tissue [1,2]. In terms of mimicking extracellular matrix, the hydrogel is known as the best candidate in tissue engineering $[2,3]$. Hydrogels have a 3D network structure of hydrophilic polymeric with a controllable mechanical property as well as the ability to release growth factors sustainably; consequently, supporting the healing of damaged tissues [2-4]. More recently, hydrogel response to changing temperature through physical crosslinking has attracted extensive studies [5]. The outstanding advantages of the temperature-responsive hydrogel are of relative ease and do not require exogenous agents that may induce immune responses inside the body [5]. Additionally, the sol-gel transition behavior of thermalsensitive hydrogel provides an injectable platform with minimal invasion compares to surgical delivery $[4,5]$.

In the modern concept of tissue engineering, hydrogel-based natural materials such as chitosan, alginate, gelatin, or collagen have been fabricated [1-3]. Among them, chitosan is of great significance for tissue regeneration [5]. Chitosan has good biocompatibility, low toxicity, and rapid biodegradability. Various studies proposed the great pharmaceutical application of chitosan, including anti-bacteria, antiinflammation, hemostasis, etc. [6,7]. Furthermore, chitosan contains abundant functional groups on its backbone; therefore, it is easy to modify or fabricate to optimize the hydrogel structure [6-8]. For example, chitosan could be co-polymerization with pluronic F127 to form the thermal sensitive hydrogel $[6,8]$. The system was considered an effective platform for drug delivery and tissue regeneration [5-7]. Poloxamer, an amphiphilic, thermo-sensitive, and FDA-approved Triblock copolymer of poly (ethylene oxide) and poly (propylene oxide), is one of the most studied platforms for the preparation of highly efficient hydrogels $[5,6,8]$. Chitosan-grafted poloxamer is widely exploited in cartilage regeneration, wound healing, burn healing, and anti-cancer drug delivery [7,9-13].

L-Arginine (Arg) is an essential amino acid that helps to prevent and treat circulatory diseases, alleviate fatigue, and stimulate the immune system. Arg is also known as endothelial nitric oxide synthase enzyme (eNOS) substrate [14], responsible for Nitric Oxide (NO) synthesis [15]. NO creates the signal for macrophage activation leading to the migration of fibroblast cells in wound healing process [14]. Along with Arg, L-Glutamic acid (Glu) is a necessary amino acid in the body, a precursor for collagen synthesis [15]. Collagen is a 
crucial protein for skin and tissue regeneration. It has been shown that the rate of collagen synthesis in tissues was completely dependent on the concentration of Arg and Glu in the micro-environment [16,17]. Therefore, Arg and Glu supplements into the hydrogel are necessary to ensure treatment outcomes of wounded areas. In this study, we develop a thermo-sensitive system of chitosan-poloxamer (CS-P407) hydrogel with Arg and Glu dual loading. The thermal-responsive behavior of the multifunctional hydrogel was evaluated by inverted tube method. The release profile with the kinetic model of Arg and Glu was also exanimated. Furthermore, the degradation of this system was investigated in the physiological medium. It is expected that CSP407 in the combination with two amino acids could be used as a promising functional wound dressing in the future clinical treatment of chronic wounds.

\section{Materials and Methods}

\section{Chemicals}

Chitosan (CS) low molecular weight 85\% deacetylated was supplied from Sigma. L-Arginine (Arg), L-Glutamic (Glu) and FMOC chloride (FMOC-Cl) were purchased from Sigma-Aldrich (USA). The cellulose dialysis membranes (molecular weight cut-off of 12-14 and $3.5 \mathrm{kDa}$ ) obtaining from Repligen were used to purify products. Mononitrophenyl formate-activated Poloxamer (NPC-P407-OH) was prepared in our previous studies at the Institute for Applied Materials Science as described [12,13]. All other chemicals were purchased from Thermo Fisher Scientific (Waltham, MA) or Fisher (USA) or of the analytical grade.

\section{Synthesis of Poloxamer P407-Conjugated Chitosan Copolymers (CS-P407)}

CS-P407 was prepared by the combination of Poloxamer P407 activated by mononitrophenyl formate with CS solution in acidic media ( $\mathrm{pH} 4-5$ ) followed by our previous report [9]. Briefly, NPC$\mathrm{P} 407-\mathrm{OH} 10^{\circ} \mathrm{C}$ was added dropwise into CS solution (mass ratio of CS: NPC-P407-OH was 1:15). After $24 \mathrm{~h}$, CS-P407 was dialyzed against distilled water using a cellulose membrane $(\mathrm{MWCO}=12-14 \mathrm{kDa})$ in 5 days and then lyophilized to obtain the final product. ${ }^{1} \mathrm{H}-\mathrm{NMR}$ and Fourier Transform Infrared Spectroscopy (FTIR) measurements were used to identify the chemical structure of copolymers.

\section{Characterizations of CS-P407 Hydrogels}

\section{Preparation of the CS-P407 Hydrogels and Bioactive Hydrogels}

The grafted copolymer CS-P407 was dissolved in DI water (PBS, DMEM) contained vials $(5,8,10,12,15$, and $20 \% \mathrm{w} / \mathrm{v})$ at $4^{\circ} \mathrm{C}$. The temperature-responsive behavior of copolymer was investigated by sol-gel transition observation with a temperature range from 15 to $50^{\circ} \mathrm{C}$, each measurement is spaced $5^{\circ} \mathrm{C}$. The temperature of the incubator was stabilized in $5 \mathrm{~min}$ before dipping the test tube. At each investigated temperature, the test tube was kept in the incubator for $10 \mathrm{~min}$ to observe the gel-sol transition behavior [16,17]. The solgel transition of the bioactive hydrogels was conducted in the same method. The three-dimensional microporous network of the hydrogel was observed by SEM.

\section{Adhesion Testing of the CS-P407 Hydrogel}

The pigskin was cleaned of fat and cut into 2 squares with dimensions of $2.5 \times 3 \mathrm{~cm}$ then soaked in 1X PBS solution ( $\mathrm{pH} 7.4$ ) for $2 \mathrm{~h}$ at $37^{\circ} \mathrm{C}$. The pigskin was then fixed on the glass slide $(25.4 \times 76.2$ $\mathrm{cm})$ and left to stabilize for about 1 hour. The hydrogel $(0.5 \mathrm{~g})$ was drenched in cold water to evenly coat the pork skin. The remaining pigskin is then placed on top of the hydrogel-coated skin. The sample was stabilized at $37^{\circ} \mathrm{C}$ then pulled with a universal tester (Portable Tension Tester MTT 1500) at $10 \mathrm{~mm} / \mathrm{min}$ until the piece of skin is separated. The value of the adhesive strength is calculated as the tensile strength at the point of the separated skin divided by the contact area of the skin. The unit of adhesive strength is measured in $\mathrm{N} / \mathrm{mm}^{2}$, then converted to $\mathrm{KPa}\left(1 \mathrm{~N} / \mathrm{mm}^{2}=1000 \mathrm{KPa}\right)$. The experiment was repeated thrice.

\section{Cytocompatibility Test of the Hydrogels}

Human fibroblasts cells (BJ (ATCC ${ }^{\oplus}$ CRL-2522 $2^{\text {mex }}$ ) were used in this study. The percentage of viable cells was determined by Sulforhodamine $\mathrm{B}$ (SRB) assay. The process was based on the guidance of Abcam. CS$\mathrm{P} 407$ hydrogel was dissolved in distilled water then irradiated with a dose of $25 \mathrm{~kJ}$ for sterilization. The copolymer solution $(100 \mu \mathrm{L})$ was evenly seeded in the culture disks with the dense of $10^{4}$ cells $/ \mathrm{mL}$, then the culture media DMEM with the supplement of $10 \%$ FBS and $1 \%$ Penicillin Streptomycin was added to reach $0.5 \mathrm{~mL}$. The untreated cells incubating with completed DMEM only was used as the control while the cell culture with $10 \mu \mathrm{L}$ water was considered as the negative control and the cell culture with $10 \mu \mathrm{L}$ Camptothecin (CPT) solution at the determined concentration $(0.2 \mu \mathrm{g} / \mathrm{mL})$ was considered as the positive control. The cells were incubated at $37^{\circ} \mathrm{C}, 90 \%$ humidity, $5 \%$ $\mathrm{CO}_{2}$ condition. At the designed time ( $4 \mathrm{~h}, 24 \mathrm{~h}, 72 \mathrm{~h}$, and $96 \mathrm{~h}$ ), the SRB kit was applied to each well. The results were recorded by ELISA at 570 $\mathrm{nm}$. The percentage of viable cells was determined by the ratio of OD value of the treated cells to untreated cells. All the experiments were repeated thrice each case with 3 replications.

\section{Release Profile and Release Kinetics of Amino Acid}

\section{CS-P407 Hydrogel Containing Amino Acid}

The fabrication of Glu/Arg loaded CS-P407 hydrogel. CS-P407 $(4.5 \mathrm{~g})$ was dissolved in $20.5 \mathrm{~mL}$ DI containing Glu/Arg at $10^{\circ} \mathrm{C}$. Copolymer solution with predetermined Glu/Arg concentration was lyophilized for further use in Glu/Arg content evaluation and in vitro release behavior. Since it is difficult to determine the content of Glu/ Arg loaded CS-P407 hydrogels, a mediated-reagent (FMOC-Cl) was used to quantify different amino acids via reactions between amino acids and FMOC-Cl [18-20]. Pure Glu/Arg was dissolved in $\mathrm{H}_{2} \mathrm{O}$ with various concentrations (in the range from 1 to $10 \mathrm{ppm}$ ). The other stock solutions were prepared by dissolving $1.237 \mathrm{~g} \mathrm{H}_{3} \mathrm{BO}_{3}$ in $100 \mathrm{~mL} \mathrm{H}_{2} \mathrm{O}$ with $\mathrm{pH}$ was adjusted to 9 by $\mathrm{NaOH} 0.1 \mathrm{M}$ and $100 \mathrm{ppm} \mathrm{FMOC-Cl} \mathrm{in}$ acetonitrile. A mixture containing 3 stock solutions as follows Glu/Arg: $\mathrm{H}_{3} \mathrm{BO}_{3}: \mathrm{FMOC}-\mathrm{Cl}=1: 1: 2(\mathrm{v} / \mathrm{v})$ was stirred at $30^{\circ} \mathrm{C}$ in $2 \mathrm{~h}$. The residual FMOC-Cl and FMOC-OH were eliminated by diethyl ether $(5 \times 5 \mathrm{~mL})$. The remaining solution was diluted to $10 \mathrm{~mL}$. UV-Vis spectra (Agilent 8453 UV-Vis Spectrophotometer) were used to determine each amino 


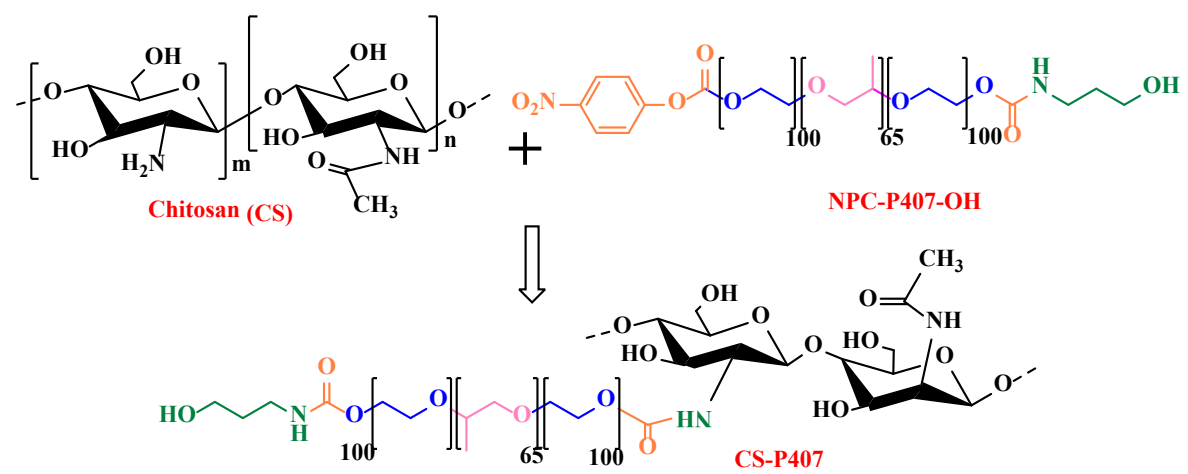

Figure 1: Synthesis of thermosensitive copolymer CS-P407

acid content at the absorb wavelength of $265 \mathrm{~nm}$. The release profiles of loading agents from the hydrogels in vitro were characterized by selfdiffusive method of Glu/Arg-loaded hydrogel contained in a cellulose membrane $(\mathrm{MCWO}=3.5 \mathrm{kDa})$. Samples $(2 \mathrm{~mL}$ hydrogel $18 \%)$ was immersed in the phosphate-buffered saline (PBS) pH $7.4(20 \mathrm{~mL})$ and shaken $(100 \mathrm{rpm})$ at $37 \pm 1^{\circ} \mathrm{C}$. At predetermined intervals ( $0 \mathrm{~h}, 1 \mathrm{~h}, 3 \mathrm{~h}$, $5 \mathrm{~h}, 7 \mathrm{~h}, 9 \mathrm{~h}, 12 \mathrm{~h}, 24 \mathrm{~h}, 36 \mathrm{~h}, 48 \mathrm{~h}$ ), the released PBS was collected and replaced by fresh PBS. The number of released agents was measured using UV-vis spectrophotometer as mentioned. The experiments were repeated 3 times. The percentage of released Glu/Arg was calculated as follows:

$$
\mathrm{Q}=\mathrm{C}_{\mathrm{n}} \mathrm{V}_{\mathrm{s}}+\mathrm{V}_{\mathrm{t}} \sum_{\mathrm{i}=1}^{\mathrm{n}-1} \mathrm{C}_{\mathrm{n}-1}
$$

Where $\mathrm{Cn}$ is the concentration of Glu/Arg in the sample, Cn-1 is the concentration of Glu/Arg released at time t, Vs is the volume of incubation medium, and $\mathrm{Vt}$ is the volume of medium replaced at time $\mathrm{t}[21]$.

The release profiles of Glu/Arg were found to be suitable for zero and first-degree equations, Higuchi, and Korsmeyer [22,23]. The mean dissolving time (MDT) value was calculated from release kinetic data using equation (Mockel and Lippold) [24].

MDT (hour) $=\left(\frac{\mathrm{n}}{\mathrm{n}+1}\right) \mathrm{k}^{-\frac{1}{\mathrm{n}}}$

Where $\mathrm{n}$ and $\mathrm{k}$ are the release exponent and the release rate constant from the Korsmeyer equation, respectively.

\section{Degradation Profiles}

Approximately $2 \mathrm{~mL}$ hydrogels $(\mathrm{Mi})$ were fabricated in vials subsequently incubated at $37^{\circ} \mathrm{C}$. The samples were prepared in distilled water and then diluted in $5 \mathrm{~mL}$ buffer PBS pH 7.4 or DMEM. After every 2 days incubation, the liquid was consecutively replaced by $5 \mathrm{~mL}$ culture media until the hydrogels had completely disintegrated. The weight of the remaining hydrogel is recorded by an electronic balance. The data profiles were expressed as the average of three measurements. The percentage weight loss is calculated as follows:

$$
\text { Weight loss }(\%)=\frac{M_{i}-M_{t}}{M_{i}} \times 100 \%
$$

$M_{i}$ : initial gel mass $(\mathrm{g}) ; M_{t}$ : the remaining gel mass $(\mathrm{g})$ and after the degraded time.

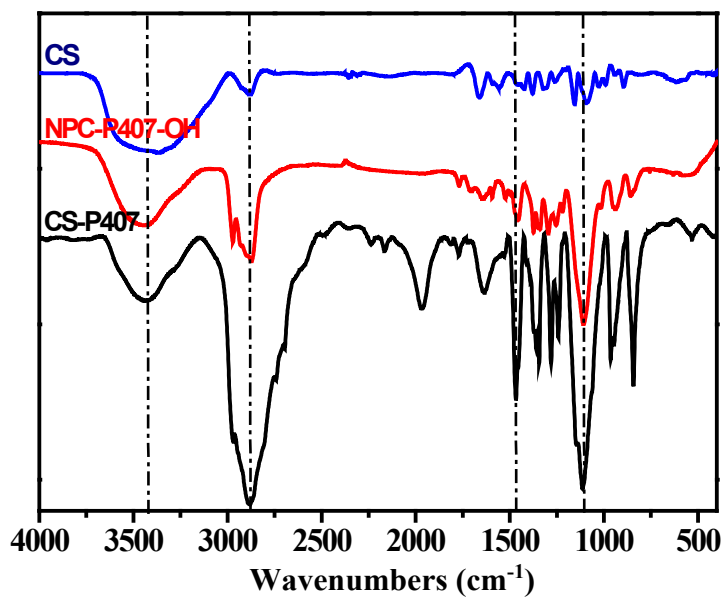

Figure 2: The FTIR spectra of P407, NPC-P407-OH, and CS-P407.

\section{Results and Discussions}

\section{Characterizations of the Amphiphilic CS-P407 Copolymers}

CS-P407 is synthesized based on the carbamate group synthesis reaction through a covalent bond between the carbonate group (NPCP407-OH) and amino group (- $\left.\mathrm{NH}_{2}\right)$ of CS (Figure 1). The ${ }^{1} \mathrm{H}-\mathrm{NMR}$ spectroscopy of CS-P407 copolymer has been mentioned in our previous research $[25,26]$. Overall, the resonance signal of Poloxamer protons and methyl, methylene and methine group exist in 3.41 $4.03 \mathrm{ppm}$ and $1.10 \mathrm{ppm}\left(-\mathrm{CH}_{3}\right.$ of PPO unit). The max resonance was achieved at $\delta=1.97 \mathrm{ppm}, \delta=2.90 \mathrm{ppm}$, and $\delta=4.64 \mathrm{ppm}$ for protons in glucosamine of CS backbone, which confirmed the structure of coconjugated compound CS-P407. The FTIR result (Figure 2) describes the spectroscopic features of standard CS in $3368.02 \mathrm{~cm}^{-1}$ wavenumber due to oscillation of $\mathrm{O}-\mathrm{H}$ bond, $1558.89 \mathrm{~cm}^{-1}$ is the oscillation of $\mathrm{N}-\mathrm{H}$ bond. The NPC-P407-OH spectroscopy shows that the peak in $2885.96 \mathrm{~cm}^{-1}$ belongs to the $\mathrm{C}-\mathrm{H}$ bond in the PEO fraction of Poloxamer P407. The peak at $1111.03 \mathrm{~cm}^{-1}$ is caused by a specific C-O linkage of Poloxamer P407. These signals also occurred in CS-P407 spectroscopy at $2890.07 \mathrm{~cm}^{-1}$ is a peak of $\mathrm{C}-\mathrm{H}$ linkage. The stretched oscillation of the $\mathrm{C}-\mathrm{O}$ bond shows a signal at $1111.88 \mathrm{~cm}^{-1}$. The peak at $1571.05 \mathrm{~cm}^{-1}$ indicates the -NH deforming signal of the amine group in CS, even though this signal doesn't exist in CS-P407 spectroscopy due to the amine group has formed a bond with $\mathrm{P} 407$ and create an amide I group with a wavenumber at around $1650.04 \mathrm{~cm}^{-1}$ [27]. To sum up, 
Ngoc Quyen Tran (2021) Dual arginine and glutamic amino acids delivery effectiveness of injectable chitosan-Poloxamer P407 towards wound healing application

the FTIR spectroscopy data shows that the peaks observed are suitable with the expected functional groups in the compound structure of conjugated copolymer.

\section{Characterizations of CS-P407 Hydrogels}

The CS-P407 hydrogel was coated with a thin layer of approximately $2 \mathrm{~mm}$ on the glass slide and the samples were allowed to dry naturally. The sample was then measured by SEM to observe the hydrogel surface structure.

SEM imaging results of Figure 3 show that the CS-P407 hydrogel has an abundant-porous structure $(1-2 \mu \mathrm{m})$ formed by the overlapping network of CS-P407 copolymers. This is the most important characteristic of the hydrogel system (scaffold) since it is not only able to absorb the fluid exudate and maintain certain water content in the wound but also allows the fibroblasts to divide and migrate. The interconnected porous structure has an impact on the supply of nutrient and gas exchange in order to maintain cellular ingrowth and retain a high amount of water, and also it offers ideal material for the Glu/Arg delivery carrier system [10].

\section{Thermal Sensitivity of CS-P407 Solution}

Obtained results of sol-gel transition investigation show that CS$\mathrm{P} 407$ solution can create gel at a relatively low copolymer concentration (above $10 \% \mathrm{wt} / \mathrm{v}$ ) at $32-37^{\circ} \mathrm{C}$ (Figure $4 \mathrm{~A}$ ). When replacing water with $\mathrm{PBS}$ buffer and DMEM cell culture media, the transition temperature is unaffected significantly. The thermosensitive hydrogel was formed via hydrophobic interaction of hydrophobic PPO domains in the CSP407 as seen in Figure 4B. The phenomenon was reported in several studies $[5,7,9,10]$.

\section{Adhesion of the Hydrogel Scaffolds}

The cohesive ability of hydrogel material to the skin surface is a critical factor in creating material for wound healing. The cohesion process will suppress plasma leakage, prevent bacterial infection, maintain gaseous exchange and provide better access to bioactive compounds in the hydrogel. The tissue adhesive experiment was conducted in porcine skin. As shown in Figure 5, the adhesivity of CS-P407 hydrogels at $15 \% \mathrm{Wt}, 18 \% \mathrm{Wt}$, and $20 \% \mathrm{Wt}$ are $5.51 \pm 0.88$ $\mathrm{KPa}, 6.62 \pm 0.7 \mathrm{KPa}$ and $5.74 \pm 0.74 \mathrm{KPa}$, respectively. This result was similar to Ji Hyun Ryu's research in 2011 (The value in this study are about 5.3 $\pm 2.6 \mathrm{KPa}$ ) [28]. At the same condition, $\mathrm{P} 407$ hydrogel exhibits a very low tissue adhesion, at which the value is $0.79 \pm 0.26$ $\mathrm{KPa}$ that is similar to a previous study $(0.72 \pm 0.32 \mathrm{KPa})$ [29].

The experiment was repeated three times independently $(n=3)$, and the errors are presented in S.E. Statistical significance of $\mathrm{p}<$ 0.001 is indicated by ${ }^{* * *}$. Non-statistical insignificance of $p \geq 0.05$ is indicated by ns.

This adhesive feature is formed by the positively charged amine groups in the CS backbone interact with the collagen matrix of porcine skin. It depends on chitosan, a polymer with strong adhesivity
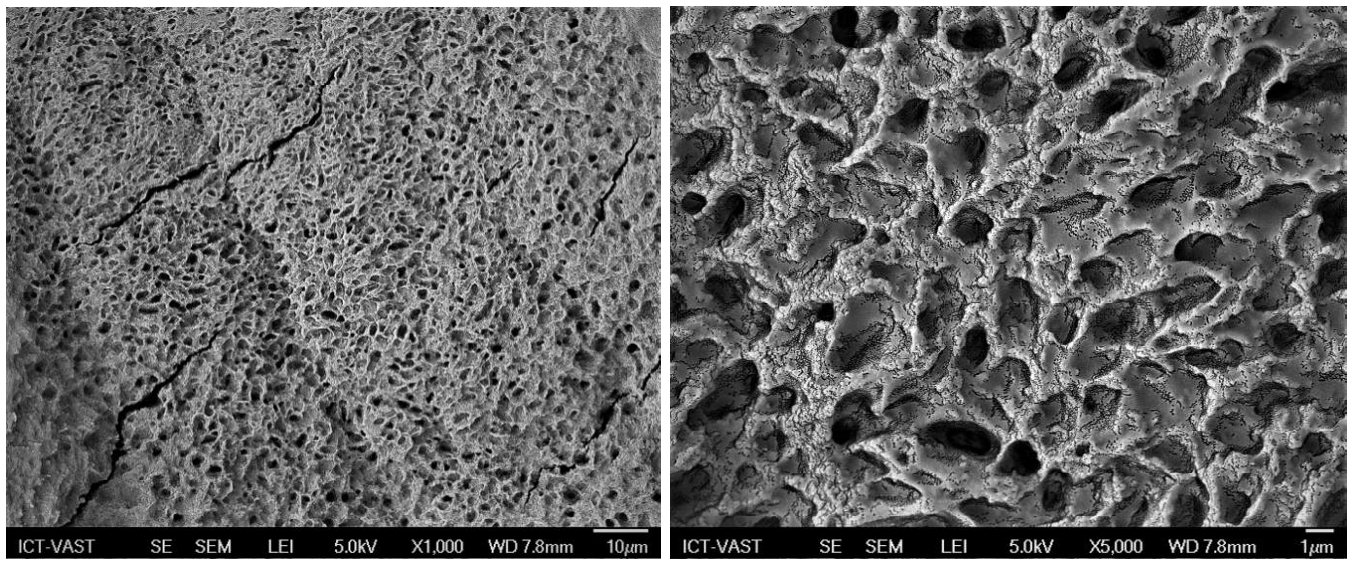

Figure 3: C-P407 hydrogel surface structure (x1000 image on the left) and (x5000 image on the right)

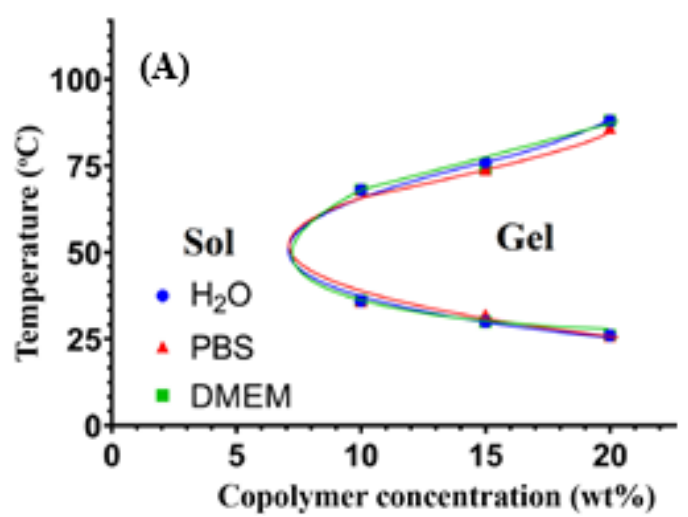

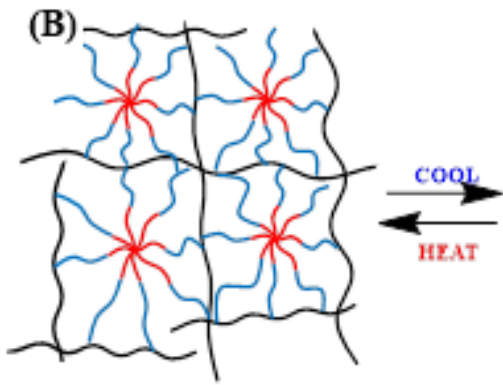

Gel state

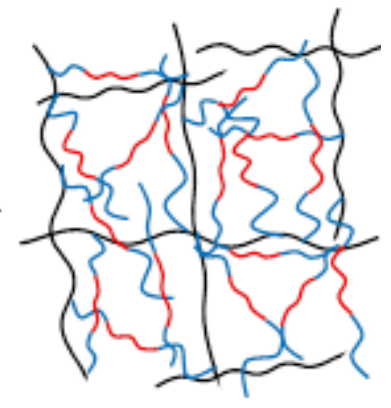

Sol state

Figure 4: The phase diagram of the sol-gel transition (A); illustration of the sol-gel transition of the CS-P407 solution (B). 


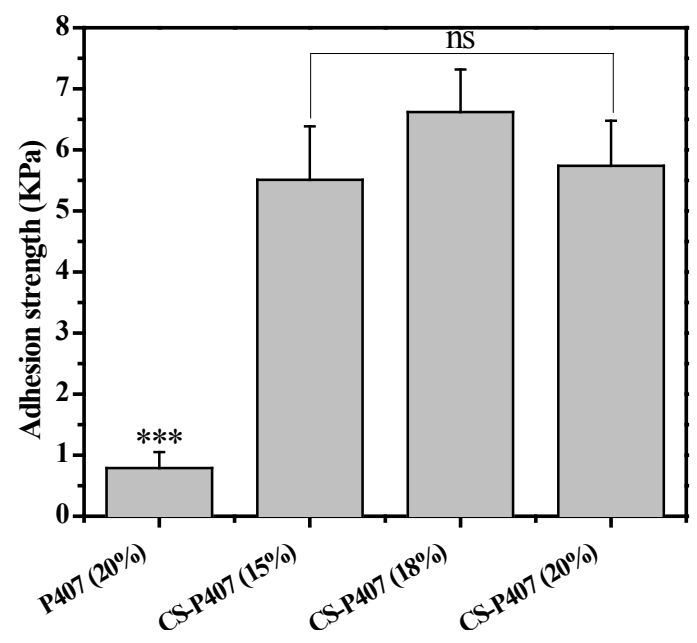

Figure 5: Adhesion strength $(\mathrm{KPa})$ of the material to the pigskin surface.

through its $-\mathrm{NH}_{2}$ groups. As a result, when attaching to the skin surface, CS-P407 can interact with the surface through hydrogen bonds, electrostatic bonds, hydrophobic interaction that make CSP407 have better adhesive intensity.

\section{Cytotoxicity of Hydrogel CS-P407}

In biomedical material, biocompatibility, or the fitness and harmlessness of the material with the human body and related physiological activities, is the primary criteria to decide its possibility for application. Fibroblasts are vital for wound regeneration, especially in the division, differentiation, and migration stage of fibrocytes at the wound surface. These cells synthesize and secrete extracellular proteins, mainly collagen, to reconstruct the extracellular matrix of connective tissue. In this research, a preliminary cytotoxicity assessment was conducted on fibroblast cell lines from human skin. According to the results in Figure 6, CS-P407 hydrogel is non-toxic for fibroblast. CPT was used at $3 \mu \mathrm{g} / \mathrm{mL}$, the fibroblast growing rate decreased $42.02 \pm 8.05 \%$ after $4 \mathrm{~h}$ of exposure and go down to $7.21 \pm$ $5.38 \%$ after 24 hours with total cell death was observed after 48 hours. The number of cells on CS-P407 increase from $106.07 \pm 3.52 \%$ after 4 hours to $136.8 \pm 5.07 \%$ after 48 hours. This proves that besides its nontoxicity, CS-P407 can improve cell growth for fibroblasts.

\section{In Vitro Amino Acid Release Profile and Release Kinetic Models}

In this study, we investigated the proliferative capacity of human fibroblasts (ATCC ${ }^{\oplus}$ CRL-2522 $2^{\mathrm{m}}$ ) of free Arg and Glu. Tested concentrations of Arg and Glu from $0-250 \mu \mathrm{M}$ and $0-500 \mu \mathrm{M}$ respectively. Figure S.1 showed that the increasing concentration of active ingredients leads to cell proliferation, proving that both Arg and Glu support cell growth and increase wound healing. Arg's support was significantly impactful on proliferation. Specially, $50 \mu \mathrm{M}$ for Arg (number of cells $=9 \times 10^{4}$ ) and $250 \mu \mathrm{M}$ for Glu (number of cells = $8 \times 10^{4}$ ) are the optimal concentration for cell proliferation. On the other hand, the excessive use of Arg and Glu also reduces cell growth. This inhibition of cell growth can be seen above $200 \mu \mathrm{M}$ for Arg and above $450 \mu \mathrm{M}$. Based on obtained data, we selected the optimal carry concentrations of Arg and Glu are $50 \mu \mathrm{M}$ and $250 \mu \mathrm{M}$, respectively, into CS-P407. These primary results are the premise to develop a hydrogel system CS-P407 to support treatment and wound healing, show great applications in medicine in general and tissue regeneration in particular.

The gelation temperature range was determined from the minimum temperature of gel formation $\mathrm{T}_{\text {gel }}$ to the temperature at which the gel begins to melt $\mathrm{T}_{\mathrm{m}}$. In Figure 7, from the measured results, it can be seen that the $\mathrm{T}_{\text {gel }}$ gelation temperature of CS-P407 carrying amino acids when mixed with distilled water is higher than that of PBS night medium and physiological DMEM medium at the same concentration. Gel forming temperature range in an aqueous medium is mostly narrower, the results are similar to that of CSP407 hydrogel. The influence of the medium on the gelation of the hydrogel can be seen. It is understood that in the medium PBS and DMEM gel has higher mechanical properties, more stable. The above results show that the gel is effective in carrying amino acids, without losing the inherent mechanical properties of the system. The CS-P407 polymer system creates a gel at $30-35^{\circ} \mathrm{C}$ and the gel melting point is above $50^{\circ} \mathrm{C}$, so it is very suitable for biomedical applications, especially wound treatment gels.

Figure 8 represents the release rate of free Glu and Arg after 12 hours achieve $100 \%$ when CS-P407/Glu, CS-P407/Arg, and CS-P407/ Glu/Arg rates are $32.53 \%, 47.28 \%$, and $65.02 \%$. After 48 hours, the
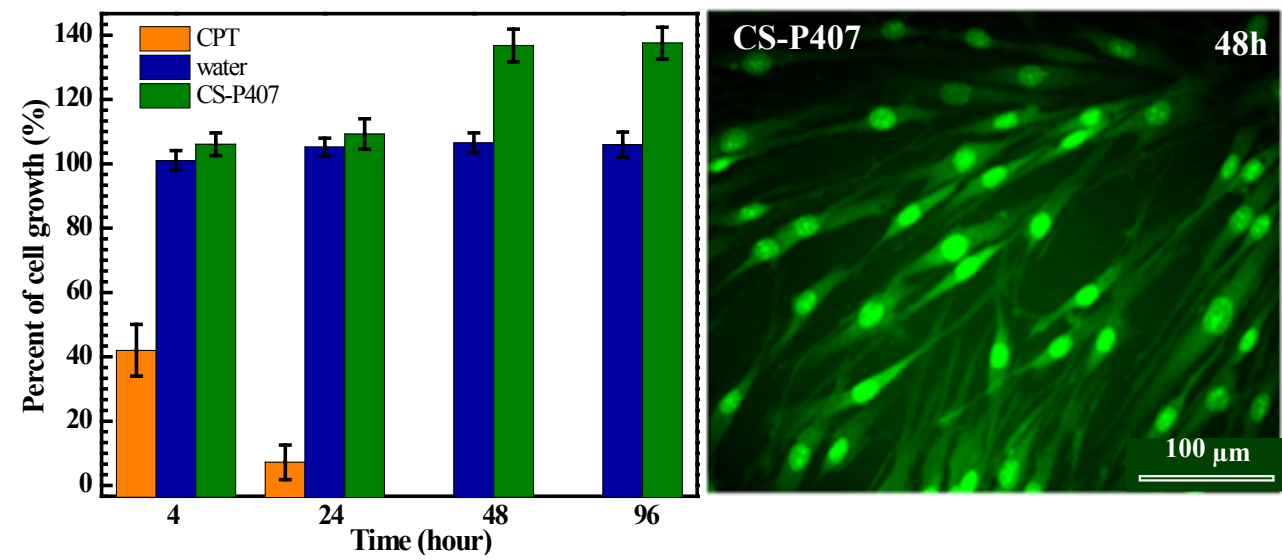

Figure 6: Percentage of BJ fibroblasts growth incubated in $0.1 \mathrm{~mL}$ of CS-P407, water (negative control) and CPT (positive controls). 


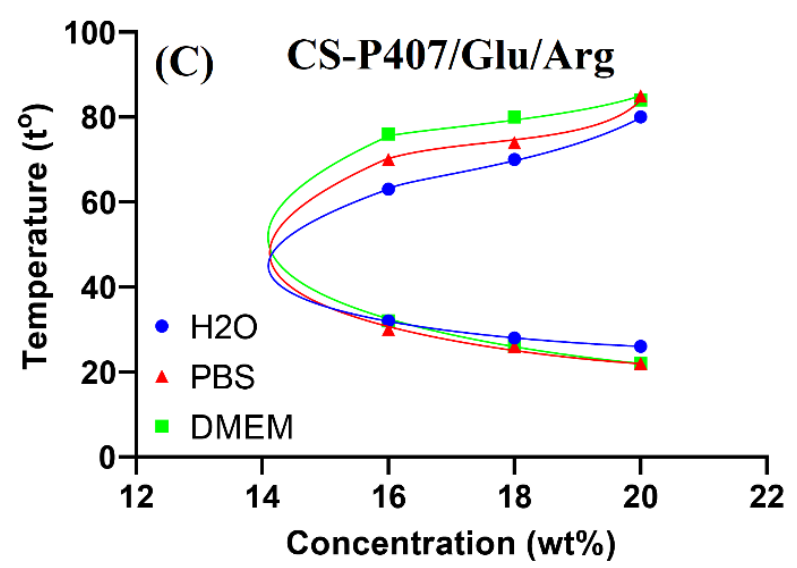

Figure 7: The phase diagram of the sol-gel transition of CS-P407/Arg (A); CS-P407/Glu (B) và CS-P407/Glu/Arg $(\mathrm{C})$

release rate at CS-P407/Glu and CS-P407/Arg reach 43.97\% and 96.83\%, respectively. The Arg release profile of CS-P407 was faster compared to Glu. This difference is according to the carboxylated form of the $2-\mathrm{COOH}$ group in Glu at $\mathrm{pH}=7.4$ has electrostatic interaction with the positively charged structure of CS-P407, which leads to a slower release rate. When both amino acids were captured in the hydrogel, their release rate reaches $80.59 \%$ after 48 hours. Thus, CS-P407 hydrogel helps decelerate the release rate of amino acids, increases their absorptivity at wounded tissue

Among all formulas investigated, the Korsmeyer-Peppas regression model has the greatest fitness $\left(\mathrm{R}^{2}=0.9155-9.8874\right)$ (Table 1 and Figure 9). With this model, the transport exponent (n) belongs to the (0.30780.5218 ) interval, indicating that the release mechanism of free amino acid and CS-P407/Glu are Fickian diffusions. With free amino acid, it is the passive diffusion, for CS-P407/Glu is due to the CS-P407 gel barrier interaction with Glu. On the other hand, with CS-P407/Arg and CS$\mathrm{P} 407 / \mathrm{Glu} / \mathrm{Arg}$, the release mechanism is non-Fickian, influenced by

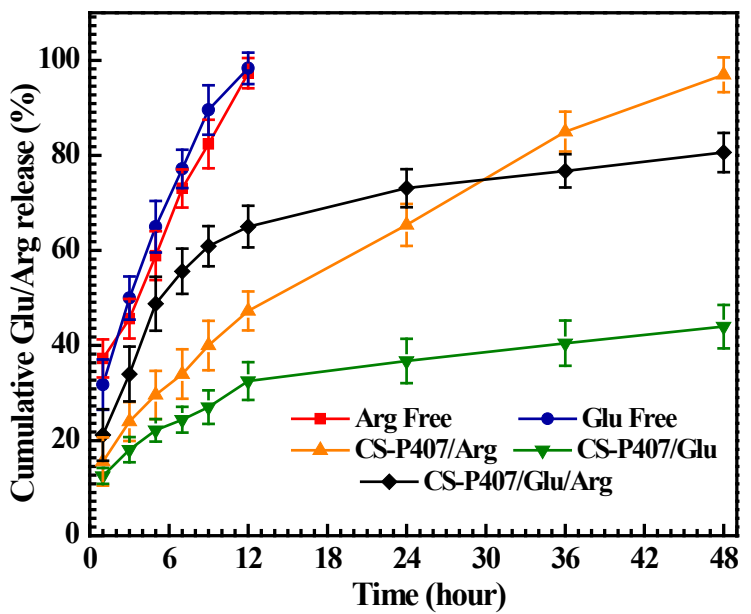

Figure 8: Release profile of amino acid (Glu/Arg) from the hydrogel CS-P407

diffusion and swelling. The rate of diffusion and swelling are the same. The rearrangement of polymer chains happens in slow progress, while the diffusion triggers some abnormal effects over time [30,31].

Table 2 shows that the MDT value of hydrogel is much higher than free amino acid, which means that amino acids have been entrapped inside the hydrogel matrix. This improves the usage efficiency of the bioactive compound with a short half-life. Thus, CS-P407 hydrogel has prominent potential in the drug distribution process in wound treatment.

\section{Degradation Profiles}

The hydrogel degradability was evaluated by gravimetric analysis of CS-P407 in PBS (pH 7.4) or DMEM solution until the hydrogel was completely degraded. Figure 10 shows that CS-P407 hydrogel had a degradation time of 10 days in PBS buffer with $\mathrm{pH}$ 7.4, which was 1.7 times longer than the P407 hydrogel sample. This shows that the combination of Poloxamer P407 with CS (which is a bio-adhesive

Table 1: Amino acid release parameters of CS-P407/Glu, CS-P407/Arg, and CS-P407/Glu/Arg hydrogel were obtained to four different mathematical models of drug release kinetics.

\begin{tabular}{|c|c|c|c|c|c|c|c|c|c|}
\hline \multirow{3}{*}{ Formulation } & \multicolumn{9}{|c|}{ Mathematical models for drug-release kinetics } \\
\hline & \multicolumn{2}{|c|}{ Zero order } & \multicolumn{2}{|c|}{ First order } & \multicolumn{2}{|c|}{ Higuchi } & \multicolumn{3}{|c|}{ Power law } \\
\hline & $k_{o}$ & $R^{2}$ & $k_{1}$ & $R^{2}$ & $\boldsymbol{k}_{H}$ & $R^{2}$ & $K$ & $n$ & $R^{2}$ \\
\hline Glu Free & 0.0981 & 0.9836 & 0.5243 & 0.9459 & 0.2911 & 0.9980 & 0.3179 & 0.4122 & 0.9972 \\
\hline Arg Free & 0.0935 & 0.9972 & 0.5182 & 0.9854 & 0.2771 & 0.9846 & 0.3339 & 0.3760 & 0.9760 \\
\hline CS-P407/Glu & 0.0121 & 0.9231 & 0.1560 & 0.8463 & 0.0799 & 0.9790 & 0.1364 & 0.3078 & 0.9920 \\
\hline CS-P407/Arg & 0.0238 & 0.9875 & 0.1805 & 0.9188 & 0.1369 & 0.9982 & 0.1422 & 0.4719 & 0.9974 \\
\hline CS-P407/Glu/Arg & 0.0233 & 0.8275 & 0.1869 & 0.7269 & 0.1611 & 0.9195 & 0.2032 & 0.5218 & 0.9155 \\
\hline
\end{tabular}

Table 2: The fit kinetic model and the MDT value of the modulation formulas.

\begin{tabular}{|c|c|c|c|c|c|c|}
\hline Formulation & Order of release & $t_{25 \%}$ (hours) & $t_{50 \%}$ (hours) & $t_{75 \%}$ (hours) & $t_{90 \%}$ (hours) & $M D T$ (hours) \\
\hline Glu Free & Fickian & 0.5583 & 3.0000 & 8.0223 & 12.4849 & 4.7056 \\
\hline Arg Free & Fickian & 0.4631 & 2.9265 & 8.6043 & 13.9741 & 5.0533 \\
\hline CS-P407/Glu & Fickian & 7.1578 & 68.0652 & 254.1572 & 459.6082 & 152.3172 \\
\hline CS-P407/Arg & Non-Fickian & 3.3073 & 14.3690 & 33.9318 & 49.9357 & 20.0140 \\
\hline CS-P407/Glu/Arg & Non-Fickian & 1.4878 & 5.6165 & 12.2164 & 17.3257 & 7.2699 \\
\hline
\end{tabular}



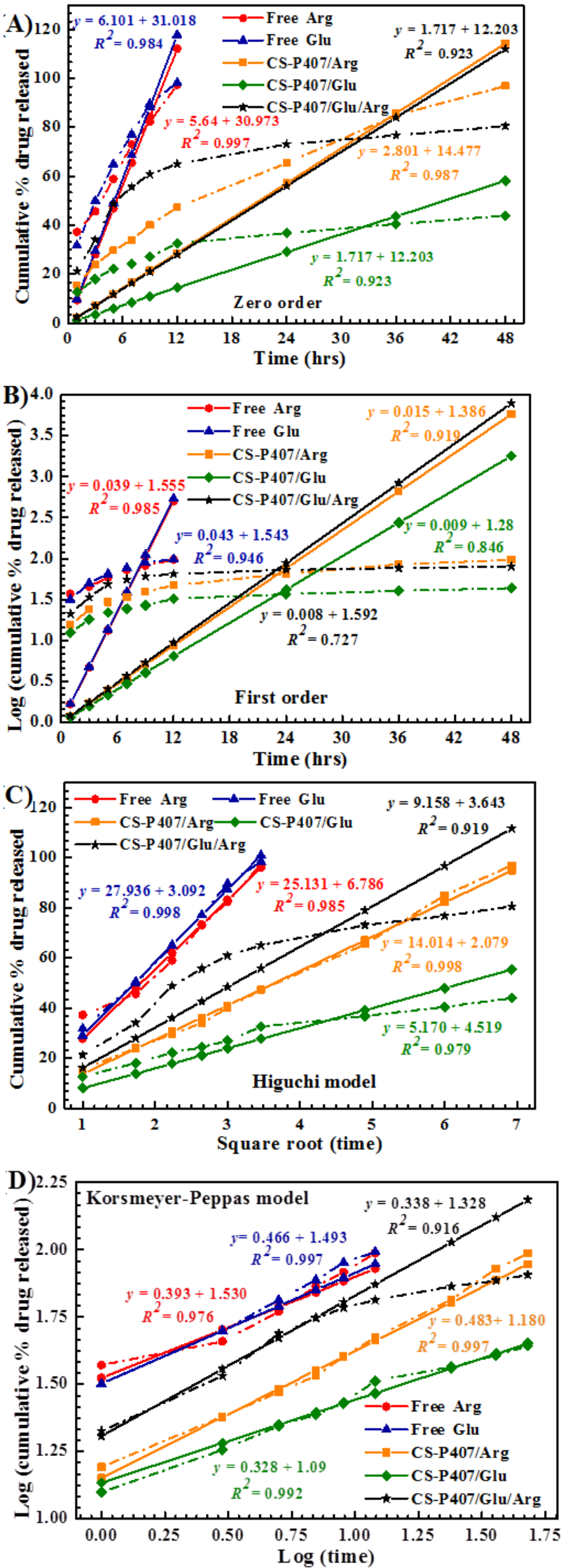

Figure 9: Release kinetics of Glu/Arg from CS-P407 fitted to four kinetic models: (A) zero-order kinetic model, (B) first-order kinetic model, (C) Higuchi model, and (D) Korsmeyer-Peppas model.
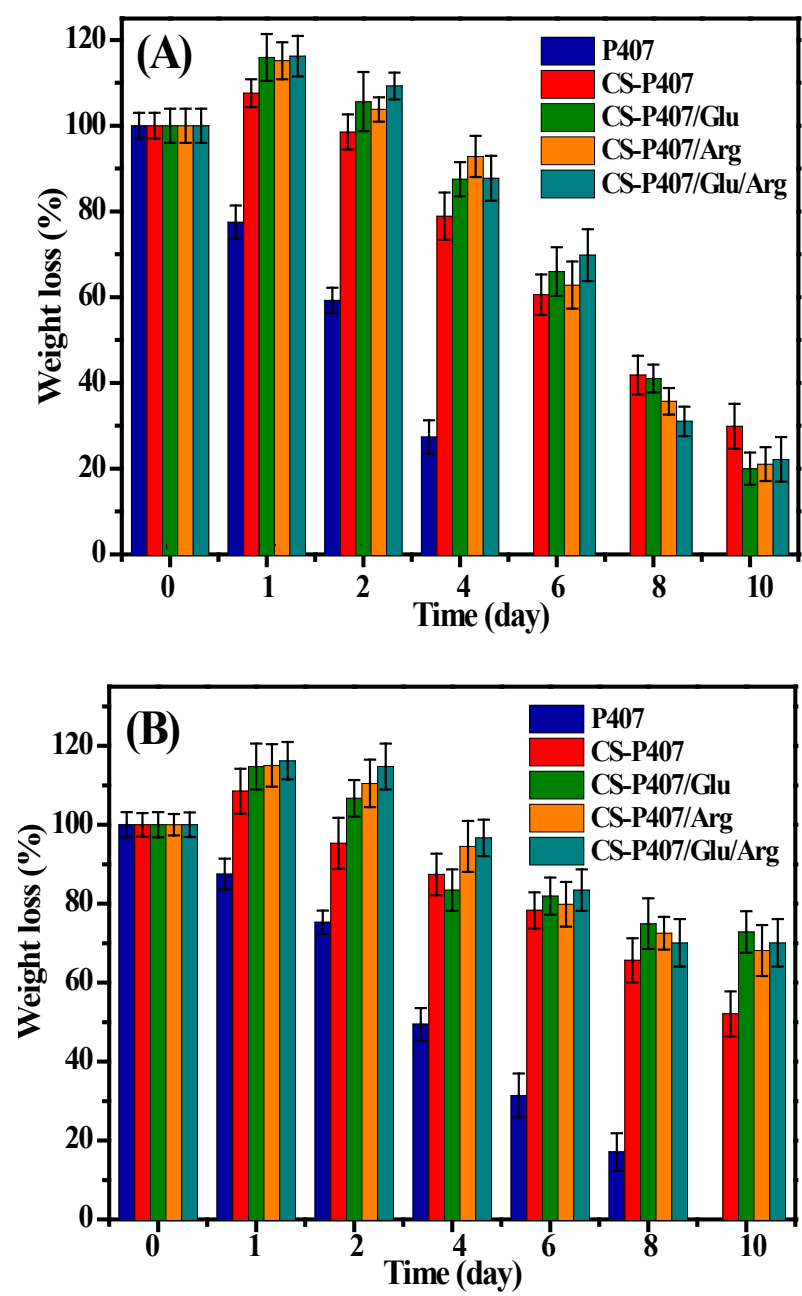

Figure 10: Degradation profile of hydrogel samples in PBS pH 7.4(A) and DMEM (B).

agent) has increased the stability of the hydrogel system. The results can be explained as the interweaving of the branched copolymer chains is increased by CS, leading to increase hydrogel stability during decomposition. Comparing degradation time in two physiological media, PBS medium pH 7.4 degraded more rapidly, when all samples have been downgraded after 12 days. DMEM medium has a longer degradation time, as the hydrogel sample remains after 16 days of investigation. Loading arginine and glutamic resulted in prolonging degradation of the bioactive hydrogels. This could be contributed by hydrogen bonding formation of amino acids and chitosan chains leading to increase the stability of the hydrogel matrix.

\section{Conclusion}

The bioactive hydrogel loading glutamic and arginine amino acid was developed. CS-P407 $18 \mathrm{wt} / \mathrm{wt} \%$ copolymer solution strongly forms hydrogel at body temperature. The system exhibits a porous structure, high tissue adhesion, and cytocompatibility which is injectable for applying minimal invasion surgery. The optimum concentrations of Arg and Glu used in the CS-P407 system to increase cell proliferation are $50 \mu \mathrm{M}$ and $250 \mu \mathrm{M}$, respectively. The CS-P407 hydrogel performed a suitable platform for controlling the delivery of these amino acids. The release behavior is affected by concentration diffusion and the 
swelling of the gel system. Arginine and glutamic encapsulation resulted in the prolonged degradation of bioactive hydrogels. The preliminary results could pave the way to apply the bioactive hydrogel in would healing.

\section{Acknowledgements}

This work was supported by the Ho Chi Minh Department of Science and Technology (number of contract 47/2019/HĐQPTKHCN).

\section{References}

1. Pramanik S, Pingguan-Murphy B, and Osman NAA (2012) Progress of key strategies in development of electrospun scaffolds: bone tissue. Science and Technology of Advanced Materials 13: 043002.

2. Wang K, Hao Y, Wang Y, Chen J, Mao L, et al. (2019) Functional hydrogels and their application in drug delivery, biosensors, and tissue engineering. International Journal of Polymer Science.

3. Le PN, Huynh CK, Tran NQ (2018) Advances in thermosensitive polymer-grafted platforms for biomedical applications. Materials Science and Engineering: C 92: 10161030. [crossref]

4. Bhattarai N, Gunn J, Zhang M (2010) Chitosan-based hydrogels for controlled, localized drug delivery. Advanced drug delivery reviews 62: 83-99.

5. Choi JH, Joung YK, Bae JW, Choi JW, Quyen T, et al. (2011) Self-assembled nanogel of pluronic-conjugated heparin as a versatile drug nanocarrier. Macromolecular research 19: 180-188.

6. Antoniraj MG, Kumar CS, Kandasamy R (2016) Synthesis and characterization of poly (N-isopropylacrylamide)-g-carboxymethyl chitosan copolymer-based doxorubicin-loaded polymeric nanoparticles for thermoresponsive drug release. Colloid and Polymer Science 294: 527-535.

7. Huynh NT, Dang LH, Pham NO, Nguyen CT, Vu MT, et al. (2019) Injectable nanocurcumin-dispersed gelatin-pluronic nanocomposite hydrogel platform for burn wound treatment. Bulletin of Materials Science, 42: 1-10.

8. Nguyen TTC, Nguyen CK, Nguyen TH, Tran NQ (2017) Highly lipophilic pluronicsconjugated polyamidoamine dendrimer nanocarriers as potential delivery system for hydrophobic drugs. Materials Science and Engineering: C 70: 992-999.

9. Dang LH, Nguyen TH, Tran HLB, Doan VN, Tran NQ (2018) Injectable nanocurcumin-formulated chitosan-g-pluronic hydrogel exhibiting a great potential for burn treatment. Journal of healthcare engineering. [crossref]

10. Pham L, Dang LH, Truong DM, Nguyen TH, Le L, et al. (2019) A dual synergistic of curcumin and gelatin on thermal-responsive hydrogel based on Chitosan-P123 in wound healing application. Biomedicine \& Pharmacotherapy 117: 109183.

11. Park KM, Lee SY, Joung YK, Na JS, Lee MC, et al. (2009) Thermosensitive chitosanPluronic hydrogel as an injectable cell delivery carrier for cartilage regeneration. Acta biomaterialia 5: 1956-1965. [crossref]

12. Hosseinzadeh H, Atyabi F, Dinarvand R, Ostad SN (2012) Chitosan-Pluronic nanoparticles as oral delivery of anticancer gemcitabine: preparation and in vitro study. International journal of nanomedicine 7: 1851-63. [crossref]

13. Nguyen VT, Nguyen TH, Dang LH, Vu-Quang H, Tran NQ (2019) Folate-conjugated chitosan-pluronic P123 nanogels: synthesis and characterizations towards dual drug delivery. Journal of Nanomaterials.

14. Fujiwara T, Kanazawa S, Ichibori R, Tanigawa T, Magome T, et al. (2014) L-arginine stimulates fibroblast proliferation through the GPRC6A-ERK1/2 and PI3K/Akt pathway. Plos one 9: e92168.

15. Aalto M, Lampiaho K, Pikkarainen J, Kulonen E (1973) Amino acid metabolism of experimental granulation tissue in vitro. Biochemical Journal 132: 663-671. [crossref]

16. Szekalska M, Puciłowska A, Szymańska E, Ciosek P, Winnicka K (2016) Alginate: current use and future perspectives in pharmaceutical and biomedical applications. International Journal of Polymer Science.

17. An D, Ji Y, Chiu A, Lu YC, Song W, et al. (2015) Developing robust, hydrogel-based, nanofiber-enabled encapsulation devices (NEEDs) for cell therapies. Biomaterials 37: 40-48. [crossref]
18. Armstrong DW, Gasper M, Lee SH, Zukowski J, Ercal N (1993) D-amino acid levels in human physiological fluids. Chirality 5: 375-378. [crossref]

19. Nguyen CT, Dang LH, Nguyen DT, Tran KP, Giang BL, et al. (2019) Effect of GA3 and Gly plant growth regulators on productivity and sugar content of sugarcane. Agriculture 9: 136.

20. Zhou W, Zhanga XY, Duana GL (2011) Liquid-Chromatography Quantitative Analysis of 20 Amino Acids after Derivatization with FMOC-Cl and Its Application to Different Origin Radix isatidis. Journal of the Chinese Chemical Society 58: 509-515.

21. Nguyen TD, Nguyen TN, Nguyen TT, Ivanov IA, Nguyen KC, et al. (2019) Nanoencapsulation enhances anticoagulant activity of adenosine and dipeptide IleTrp. Nanomaterials 9: 1191.

22. Kuksal A, Tiwary AK, Jain NK, Jain S (2006) Formulation and in vitro, in vivo evaluation of extended-release matrix tablet of zidovudine: influence of combination of hydrophilic and hydrophobic matrix formers. AAPS pharmscitech 7: E1-E9. [crossref]

23. Sahu A, Kasoju N, Goswami P, Bora U (2011) Encapsulation of curcumin in Pluronic block copolymer micelles for drug delivery applications. Journal of biomaterials applications 25: 619-639. [crossref]

24. Roni MA, Kibria G, Jalil RU (2009) In vitro studies of controlled release alfuzosin matrix tablets prepared with ethylcellulose and hydroxypropyl methylcellulose. Iranian Journal of Pharmaceutical Sciences.

25. Dang LH, Vu MT, Chen J, Nguyen CK, Bach LG, et al. (2019) Effect of ultrasonication on self-assembled nanostructures formed by amphiphilic positive-charged copolymers and negative-charged drug. ACS Omega 4: 4540-4552.

26. Nguyen DH, Nguyen VT, Nguyen CK, Nguyen TH (2016) Green processing of thermosensitive nanocurcumin-encapsulated chitosan hydrogel towards biomedical application.

27. Nguyen TBT, Dang LH, Nguyen TT, Tran DL, Nguyen DH, et al. (2016) Green processing of thermosensitive nanocurcumin-encapsulated chitosan hydrogel towards biomedical application. Green Processing and synthesis 5: 511-520.

28. Ryu JH, Lee Y, Kong WH, Kim TG, Park TG et al. (2011) Catechol-functionalized chitosan/pluronic hydrogels for tissue adhesives and hemostatic materials. Biomacromolecules 12: 2653-2659. [crossref]

29. Lee Y, Chung HJ, Yeo S, Ahn CH, Lee H, et al. (2010) Thermo-sensitive, injectable, and tissue adhesive sol-gel transition hyaluronic acid/pluronic composite hydrogels prepared from bio-inspired catechol-thiol reaction. Soft Matter 6: 977-983.

30. Mircioiu C, Voicu V, Anuta V, Tudose A, Celia C, et al. (2019) Mathematical modeling of release kinetics from supramolecular drug delivery systems. Pharmaceutics 11: 140. [crossref]

31. Shaikh HK, Kshirsagar RV, Patil SG (2015) Mathematical models for drug release characterization: a review. World J. Pharm. Pharm. Sci 4: 324-338.

\section{Citation:}

Tran NQ, Nguyen DT, Dang LH, Nguyen BT (2021) Dual arginine and glutamic amino acids delivery effectiveness of injectable chitosan-Poloxamer P407 towards wound healing application. J Pharmacol Pharm Res Volume 4(3): 1-9. 


\section{Appendix A. Supplementary data}
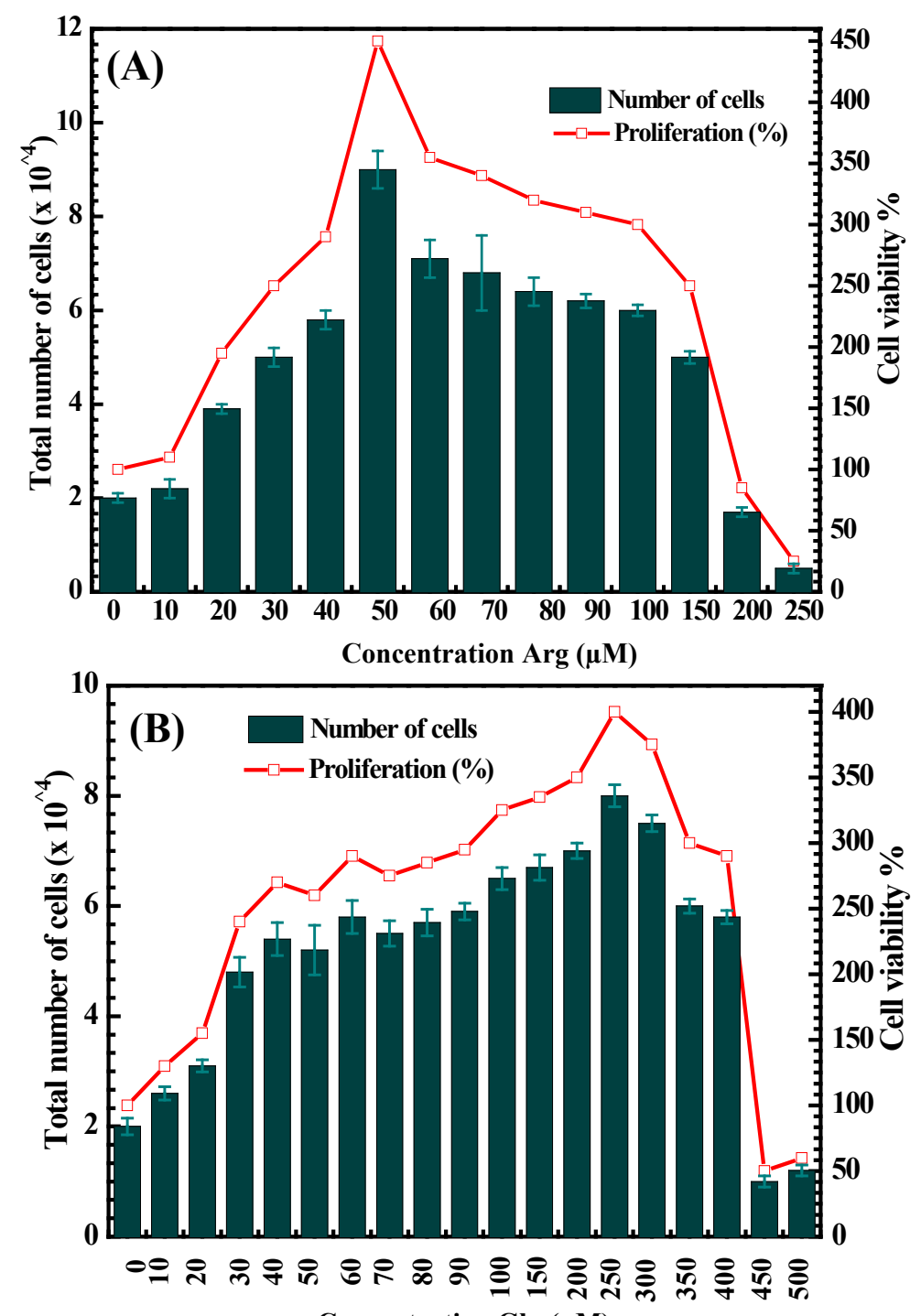

Figure S.1. Concentration of amino acids versus fibroblast proliferation: Arg (A) and Glu (B). 